\title{
La responsabilité sociale de l'écrivain. Denis de Rougemont et le PEN Club dans les années 1970
}

\section{Nicolas Stenger}

\section{(2) OpenEdition}

1 Journals

Édition électronique

URL : https://journals.openedition.org/edl/1848

DOI : $10.4000 /$ edl. 1848

ISSN : 2296-5084

Éditeur

Université de Lausanne

\section{Édition imprimée}

Date de publication : 15 décembre 2019

Pagination : 49-70

ISBN : 978-2-940331-72-7

ISSN : 0014-2026

\section{Référence électronique}

Nicolas Stenger, «La responsabilité sociale de l'écrivain. Denis de Rougemont et le PEN Club dans les années 1970 ", Études de lettres [En ligne], 311 | 2019, mis en ligne le 15 décembre 2021, consulté le 16 décembre 2021. URL : http://journals.openedition.org/edl/1848; DOI : https://doi.org/10.4000/edl. 1848

Ce document a été généré automatiquement le 16 décembre 2021.

(c) Études de lettres 


\title{
La responsabilité sociale de l'écrivain. Denis de Rougemont et le PEN Club dans les années 1970
}

\author{
Nicolas Stenger
}

1 Quelles raisons poussèrent un intellectuel mondialement connu, habitué des grandes rencontres européennes et internationales, à prendre les rênes, entre 1970 et 1976, d'une petite association régionale d'écrivains tel que le centre suisse romand du PEN Club? En dépit d'une longue histoire, ponctuée aujourd'hui de commémorations (70 ans du centre suisse romand en 2019, bientôt 100 ans du PEN International en 2021), le PEN Club, ou plus simplement le PEN - acronyme de "Poets, Essayists, Novelists » faisant référence au terme anglais pen (la plume) - demeure de fait peu familier du grand public, comme des historiens ${ }^{1}$.

2 Né au lendemain de la Première Guerre mondiale, le PEN incarne d'abord une volonté de réconciliation entre les nations européennes par le maintien de liens et d'échanges littéraires, indépendamment des idéologies et des régimes politiques. Parmi les principes énoncés dans sa charte figure également le respect de la liberté d'expression et la nécessité d'une « libre critique des gouvernements et des institutions ». C'est aussi au sein du PEN que fut créé en 1960 un " comité des écrivains en prison », qui constitue en quelque sorte le précurseur d'Amnesty International ${ }^{2}$. De fait, si le centre suisse romand était une association locale, la « visée spécifique » du PEN était « universelle », comme le rappelait Denis de Rougemont lors d'un discours prononcé après cinq ans de présidence: "Nous avons tous besoin de racines et de mobilité, d'une patrie bien concrète et d'ouverture au monde ». Il en allait de même pour la littérature :

Pas question de nous réduire à des racines : nous ne sommes pas des légumes! Il existe un légume qui est tout racine, il a une mauvaise réputation en littérature : le navet. Pas question non plus de n'appartenir à aucune communauté locale ou régionale d'aucune sorte: ce serait une fuite devant les engagements civiques sans lesquels pas de réalisation de la Personne $e^{3}$.

Cette insistance sur l'appartenance à une communauté locale ou régionale et sur le civisme témoigne de l'attention que porte l'auteur dans toute son œuvre à la question 
de la responsabilité sociale de l'écrivain. Le PEN lui permit, entre autres choses, de réaffirmer cette nécessité, vitale à ses yeux, de l'engagement.

\section{Naissance d'une collaboration}

\section{1. «Parlons poésie... »}

4 Apparemment secondaire si on la compare à la durée de son engagement fédéraliste européen, l'implication de Rougemont dans les activités du PEN au début des années 1970 n'en est pas moins cohérente avec l'évolution de son parcours. Rappelons qu'avant de prendre la présidence du centre suisse romand, Rougemont avait été à la tête d'une autre association d'intellectuels dont il faut dire un mot pour comprendre le contexte de son engagement au PEN. De 1950 à 1966, l'écrivain présida en effet le comité exécutif du Congrès pour la liberté de la culture, organisation d'intellectuels libéraux aux ramifications mondiales ${ }^{4}$. En 1966, le Congrès entra dans une phase de réorganisation suite aux révélations de son financement par la Central Intelligence Agency : principal repreneur, la Fondation Ford écarta Rougemont au profit de Raymond Aron, qui présida le Congrès pendant quelques mois, avant d'en démissionner à son tour en 1967. Ce fut alors le poète Pierre Emmanuel qui, avec Shepard Stone, un transfuge de la Fondation Ford, allait diriger ce qui s'appellerait désormais l'Association internationale pour la liberté de la culture ${ }^{5}$. Pierre Emmanuel tenta à plusieurs reprises d'associer Denis de Rougemont à ses activités, sans succès, l'écrivain ayant définitivement pris ses distances avec cet héritier du Congrès, comme en témoigne une lettre de mai 1970 :

Mon cher Pierre, [...] je suis vivement touché par la manière que vous avez de m'inviter [...]. Mais ce n'est pas encore assez pour me convaincre d'aller jouer devant un Comité plus ou moins pressé par son ordre du jour le rôle d'un Nestor écarté des affaires sérieuses (sociologiques, américaines) et qui revient dire qu'il avait raison. [...] Non, je n'irai pas mettre mon vin vieux dans ces outres en plastic. Je préférais encore le bon vieux CIA qui nous foutait royalement la paix, à la Rand Corporation et autres gadgets intellectuels. Sauvez-vous de cette race perverse! Et parlons poésie ${ }^{6}$.

«Parler poésie », retrouver la compagnie des écrivains, fuir le «sociologisme » qui, à son goût, envahissait désormais l'agenda de l'Association internationale pour la liberté de la culture... Il n'est pas étonnant que Denis de Rougemont fût ouvert, en 1970, à l'idée de s'investir dans le centre suisse romand du PEN, sachant que certains de ses objectifs rejoignaient ceux du Congrès où il avait milité à l'origine, notamment le soutien aux écrivains et prisonniers politiques. La mobilisation du PEN, en 1970, pour défendre la candidature de Rougemont au prix Nobel de littérature acheva certainement de convaincre l'écrivain de participer aux destinées de l'association?

\subsection{Le centre suisse romand du PEN en 1970}

Dans quel état se trouvait le centre suisse romand du PEN quand l'écrivain en prit la présidence en 1970 ? La situation n'était pas brillante si l'on en croit une lettre que Suzanne Deriex, alors sa secrétaire, adressa à Rougemont le 30 avril 1970 en guise de premier contact :

Pas de président ; un comité fantomatique, qui ne s'est pas renouvelé depuis la nuit des temps et dont les quelques membres survivants se cramponnent à leur 
fonction; une liste de membres enfin étrangement hétéroclites: quelques sots nuisibles, plusieurs médiocres satisfaits mettent en fuite les écrivains authentiques qui font partie du PEN ou qui pourraient y adhérer et qui préfèrent ainsi choisir un autre lieu de rencontre ou d'action ${ }^{8}$.

7 Quels étaient ces autres lieux de rencontre? C'était tout d'abord la Société suisse des écrivains (SSE) ${ }^{9}$, « ce syndicat aux mamelles intarissables, qu'ils traient et dénigrent » ironisait encore Suzanne Deriex dans sa lettre. C'était ensuite le groupe d'Olten, formé de dissidents de la $\mathrm{SSE}^{10}$. Au niveau international, c'était enfin la COMES, qui venait d'être dissoute en 1969 suite à l'entrée des chars du Pacte de Varsovie en Tchécoslovaquie, et où l'on retrouvait jusque-là des poètes et écrivains comme Philippe Jaccottet et Maurice Chappaz, lesquels avaient été attirés par les possibilités de contacts qu'offrait cette association avec des écrivains situés de l'autre côté du rideau de fer. Rappelons que la COMES avait été créée en 1958 afin d'offrir une alternative au PEN International qui, en dépit de son caractère apolitique, avait «le handicap de passer pour anticommuniste aux yeux des Soviétiques »; de fait, l'originalité de la COMES venait «de ce que des écrivains soviétiques y ont officiellement adhéré » ${ }^{11}$. Sa dissolution en 1969 eut pour conséquence de poser à nouveau la question de la participation des Soviétiques aux activités du PEN, à travers le projet de création d'un PEN russe et l'adhésion des membres de l'Union des écrivains soviétiques ${ }^{12}$.

Outre un besoin de renouvellement de ses membres et de son comité, le budget du centre suisse romand était un autre sujet de préoccupation. Son montant, dérisoire au début des années 1970, entravait de fait la bonne marche de l'association : en avril 1971, on comptait à peine plus de 1'300 francs dans les caisses ${ }^{13}$. Une demande de subvention de 10'000 francs fut adressée à Pro Helvetia, en vain - le manque de coopération entre les différentes associations suisses d'écrivains étant l'un des motifs allégués par cette fondation pour refuser de soutenir le $\mathrm{PEN}^{14}$. C'est finalement grâce à un legs de $10^{\prime} 000$ francs obtenu via la sœur de Berthe Vulliemin, romancière vaudoise alors récemment décédée, et ancienne vice-présidente du centre romand, que le PEN put assurer son avenir immédiat à partir de $1972^{15}$.

\subsection{Les activités du centre suisse romand au début de la présidence Rougemont}

9 Renouveler le PEN : tel était le premier objectif de Rougemont quand il prit la présidence du centre suisse romand. 28 nouveaux membres furent ainsi cooptés entre 1971 et 1974, dont Maurice Chappaz et Philippe Jaccottet, mais aussi Jacques Mercanton, Alexandre Voisard, S. Corinna Bille, ainsi que la poétesse Anne Perrier, Albert Mermoud, alors président de la Cinémathèque suisse, ou encore Vladimir Dimitrijević, directeur des éditions L'Âge d'Homme ${ }^{16}$. Comme souvent, ces adhésions s'accompagnaient d'un investissement plus ou moins concret et suivi, selon les cas, dans les activités de l'association. Plusieurs témoignages montrent que Maurice Chappaz était trop accaparé par son œuvre, tandis que l'acceptation de Jacques Mercanton parle d'elle-même :

Je dois avouer que je n'ai jamais saisi l'utilité de ces sociétés d'écrivains, qui nourrissent de vaines querelles et des rivalités illusoires. Mais, par esprit de solidarité, je suis prêt à donner mon adhésion à celle dont vous me parlez. Ce qui, hélas, n'engage à rien ${ }^{17}$. 
L'autre souci de Denis de Rougemont, exprimé à plusieurs reprises dans les procèsverbaux des assemblées générales, était le manque de relations entre les différents centres suisses du PEN. On aboutit parfois à des situations absurdes : c'est ainsi que le président du PEN International s'adressa directement au président du PEN zurichois, Werner Weber, pour lui demander de se mettre en relation avec le PEN romand ${ }^{18}$, ce dernier essayant en vain de nouer des liens avec ses homologues en Suisse alémanique ${ }^{19}$. Les archives témoignent d'une faible activité des centres de Zurich et de Bâle, très anticommunistes à l'époque et hostiles par principe à l'existence de centres du PEN en Europe de l'Est ${ }^{20}$. De son côté, Rougemont préconisait de dissoudre ces PEN "fossilisés », et de créer un seul PEN suisse multilingue; ou bien de reformer un seul centre en Suisse alémanique ${ }^{21}$. Ce n'est qu'à partir de 1979 que l'activité du PEN en Suisse alémanique allait connaître un nouvel essor, avec la création du DeutschSchweizer PEN Zentrum à Bâle.

Si les activités du centre romand paraissent relativement modestes en Suisse, la présidence Rougemont fut en revanche marquée par une présence accrue aux activités internationales du PEN. À titre d'exemple, pour la période 1970-1974, le centre suisse romand fut représenté à tous les comités exécutifs internationaux, sauf un, ainsi qu'aux tables rondes organisées par le PEN autour de différents thèmes littéraires ${ }^{22}$. L'activité du centre suisse romand se manifesta également par différents types d'interventions, qu'il s'agisse de proposer des mesures de réorganisation (comme la marginalisation des centres PEN en sommeil), de traduire en français les brochures produites par le secrétariat international ${ }^{23}$, ou encore de soutenir, par des actions individuelles ou conjointes, les écrivains persécutés.

\section{L'écrivain et l'engagement}

12 À l'issue d'une table ronde organisée par le PEN en 1972, Rougemont rédigea un important essai intitulé Responsabilité de l'écrivain dans la société européenne d'aujourd'hui. Ce texte, qui synthétise et reformule des réflexions sur l'engagement développées dès ses premiers écrits, nous permet d'examiner la conception du rôle et du travail de l'écrivain que Rougemont souhaitait défendre auprès de ses pairs. Publié en 1973 par Pro Helvetia, traduit en allemand et en anglais, il fut diffusé dans tous les centres nationaux et locaux du PEN ${ }^{24}$.

Comment Rougemont envisageait-il la notion d'engagement ${ }^{25}$ ? Pour en trouver les premières définitions, il faut remonter aux années 1930, quand les gloses se multiplièrent autour des écrits de Julien Benda, lequel soutenait que la seule œuvre digne de l'intellectuel était - sauf à trahir sa fonction - celle de « défendre les valeurs éternelles et désintéressées comme la justice et la raison ${ }^{26} »$, sans se mêler de prendre position dans les débats de son temps. Benda souhaitait préserver l'intégrité de l'activité du penseur qu'il inscrivait dans la tradition des "grands patriciens de l'Esprit", tels Érasme, Malebranche, Spinoza...: cette activité était pour lui essentiellement spéculative. Au nom d'un "opportunisme de la vérité », Rougemont affirmait au contraire que toute pensée n'est valable qu'à partir du moment où elle quitte le domaine des « idées pures " pour épouser le réel, à partir du moment où elle est engagée, ou encore "incarnée $»^{27}$. Le concept d'engagement procédait, comme en d'autres domaines de la pensée de Rougemont, de sa foi chrétienne (dominée par les figures de Søren Kierkegaard et de Karl Barth) et de sa définition de la personne, à la 
fois «libre et responsable». Au sein des groupes personnalistes Esprit et L'Ordre nouveau, Rougemont était l'un des plus ardents défenseurs de cette dialectique penséeaction: loin d'échapper à l'Histoire et au monde, la littérature et la philosophie devaient être porteuses de solutions sur la société et la politique, leurs fondements et leurs pratiques.

Cette préoccupation était centrale dès les premiers textes de jeunesse. Voici une phrase typique des années vingt: «Écrire, pas plus que vivre, n'est aujourd'hui un art d'agrément $\aleph^{28}$. Ou encore, quelques années plus tard : « Il s'agirait, au fond, d'amener la pensée à la plus insistante vénération du réel $»^{29}$. Une telle conception du travail de l'écrivain, opposée à l'idéalisme prétendu de ses ainés, donnait invariablement aux essais de Rougemont un ton tranchant, ce dernier précisant que la littérature personnaliste serait « franchement doctrinaire et polémique ${ }^{30}$ ». Privilégier la doctrine et la polémique ne signifiait cependant pas que le travail du style devait en pâtir. L'enjeu était autant éthique qu'esthétique. Dans Penser avec les mains, Rougemont définissait ainsi un "style né de la seule passion de s'engager » :

Que chaque phrase indique la volonté d'atteindre un but, dont la nature commande le choix des mots, le rythme, les figures. Que chaque phrase implique ce but, et le désigne par son allure même. Que le style s'ordonne à sa fin et non plus à de bons modèles. Et qu'il rappelle à la situation, au lieu de rappeler des sources. Que nos écrits figurent les microcosmes de cet ordre nouveau qu'ils revendiquent. Qu'ils illustrent, dans leur structure, visible ou secrète, la dialectique joyeuse de la personne en acte. Que celui qui s'engage dans leur lecture éprouve de tout son être la présence d'une réalité éthique immédiate à chaque progrès du discours et qu'il n'en sorte pas intact ! !1 $^{31}$

15 La littérature dans son ensemble, de Gide aux surréalistes, faisait les frais d'une critique de «l'acte gratuit » ${ }^{32}$. L'engagement personnaliste était ainsi conçu comme un rappel permanent à la responsabilité, une manière de se confronter au monde, tout en inventant de nouvelles formes institutionnelles et politiques, "à hauteur d'homme». Par ailleurs, bien que solidement ancrée dans son temps, la réflexion intellectuelle que Rougemont promouvait dans ses écrits ne devait en aucun cas s'inféoder aux partis politiques :

Pour qu'une pensée s'engage dans le réel, il ne faut pas et il ne saurait suffire qu'elle se soumette à des réalités dont elle ignore ou répudie la loi interne : la tactique d'un parti, par exemple. Ce n'est pas dans l'utilisation accidentelle et partisane d'une pensée que réside son engagement. C'est au contraire, dans sa démarche intime, dans son élan premier, dans sa prise sur le réel et dans sa volonté de le transformer, donc finalement de le dominer ${ }^{33}$.

Dans son essai de 1973, Rougemont soulignait à quel point ce débat sur l'engagement, exacerbé au $\mathrm{xx}^{\mathrm{e}}$ siècle, aurait été incompréhensible aux temps anciens - ceux du mythe et de l'épopée - où la responsabilité sociale de l'auteur était une donnée de fait, qu'il eût été inconcevable de mettre en question :

De la Bhagavad-Gita à la Quête du Graal, de la tragédie d'ÆEdipe à celle de Tristan, toutes les grandes œuvres de la littérature mondiale [...] naissent et vivent en symbiose avec une communauté, en expriment les finalités, les règles instituantes, et les fatalités ${ }^{34}$.

17 Ce n'est qu'à partir du XII ${ }^{\mathrm{e}}$ siècle, selon Rougemont, en raison de la prise en main de la fonction politique ${ }^{35}$ par l'Église catholique et l'État national, que naquit progressivement en Europe une littérature autonome, distincte du social et du mythe qui en assurait la cohésion. Ce processus de démystification et de dissociation («nos 
littératures se sont progressivement dessaisies de leur mission créatrice de communauté ${ }^{36}$ ) rendit possible dès lors une réflexion et une pratique de l'engagement de l'écrivain, non plus considéré comme un fait tenu pour acquis, mais comme un problème à penser et à résoudre. Des exemples célèbres orientaient Rougemont dans sa réflexion sur les diverses formes de l'engagement, de Dante à Nietzsche, en passant par Voltaire et Rousseau ${ }^{37}$. Mais c'est essentiellement à travers l'analyse de ses contemporains, André Breton, et surtout Jean-Paul Sartre, que Rougemont allait de nouveau préciser sa conception de l'engagement.

De fait, c'est Sartre qui avait véritablement popularisé l'idée après la guerre, notamment dans le texte "Qu'est-ce que la littérature?", publié en 1948, dont Rougemont entreprit la critique dans son essai de 1973. L'essentiel du désaccord tournait autour de la confusion, entretenue par Sartre selon Rougemont, entre engagement politique et littéraire. Sartre, qui en appelait constamment à la Révolution, considérait que ce n'était nullement l'écrivain - marqué pour l'éternité par son origine bourgeoise - mais le peuple qui devait en constituer le vecteur principal. Préoccupé par la question du divorce entre l'écrivain et son public, il établissait une distinction entre public réel (les bourgeois) et public virtuel (les ouvriers), en vertu de laquelle il pouvait «feindre d'écrire pour des lecteurs qui ne le lisent pas et faire semblant d'ignorer qui le lit vraiment $»^{38}$. Le problème était quasi insoluble, car pas plus que d'autres, Sartre n'a réussi à toucher véritablement le peuple, les ouvriers; tout au plus a-t-il produit un discours sur le peuple, lu par les bourgeois qu'il détestait tant et non par ce dernier qu'il chérissait. Bien qu'attaché à la restauration d'une « commune mesure » ${ }^{39}$ entre la pensée et l'action, entre la culture et le peuple, Rougemont n'en concluait pas pour autant que «le sort de la littérature était lié à celui de la classe ouvrière ${ }^{40}$, étant donné qu'il ne raisonnait jamais en termes de classes :

Il nous faut dire que c'est l'homme en tant qu'homme - et pas seulement le non-

bourgeois - qui pâtit du désordre établi ${ }^{41}$.

Comme le souligne l'historien Antoine Prost, cette position n'était cependant guère tenable «dans le contexte de la Libération et la force de l'engagement communiste influença tous les autres engagements au point qu'on a pu confondre, pendant quelques années, engagement et engagement au service de la classe ouvrière ${ }^{42}$.

S'il en appelait constamment à l'action, Rougemont invitait à faire preuve de réalisme et d'humilité quant au sens et à l'étendue souhaitables de l'engagement de l'écrivain :

Responsable est celui qui peut dire, dans une situation donnée : j'en réponds. Mais de quoi l'écrivain comme tel peut-il répondre, sinon de son œuvre elle-même, de sa pensée et de son style ?33

Or, à sa façon, Sartre brouillait la frontière entre engagement politique et intellectuel, en traitant de "moralistes» tous ceux qui condamnaient l'activisme partisan. Il reprochait à Rougemont, " cet Européen », qualifié « d'homme doux, bien élevé et, pardessus le marché, un Suisse », de discourir dans le vide " au nom du droit des gens » et d'occulter le problème de la violence réelle qu'impliquait le jeu des forces politiques ${ }^{44}$. À l'inverse, la conception sartrienne de la praxis n'était pour Rougemont qu'une manière de sortir du rôle d'écrivain et de penseur. Ainsi à propos des surréalistes, à qui Sartre reprochait de «noircir beaucoup de papier » sans jamais rien détruire " pour de vrai »:

Sans insister sur le fait, trop évident, que le PC n'a rien cassé jusqu'ici [...], on relèvera que l'écrivain qui détruirait pour de vrai, selon Sartre, ne pourrait le faire par l'écriture mais par l'application de ce qu'il a écrit à la réalité matérielle du 
physique, donc en cessant d'être écrivain, en reniant sa fonction propre, tel un poteau indicateur qui déciderait de faire lui-même le chemin et cesserait aussitôt d'être utile. La vraie révolution n'est pas celle qui détruit, et la production de valeurs et d'un modèle neuf de société n'a jamais été le fait du militant de base (électeur, manifestant, gréviste ou franc-tireur), mais dans tous les cas que l'on connaît, d'hommes qui ont écrit et même beaucoup écrit. Ce n'est pas parce qu'il n'a rien cassé pour de vrai que Breton n'était pas engagé, mais parce que sa pensée ne fut jamais en puissance d'action politique et demeure purement subversive (comme le fait de descendre dans la rue un revolver dans chaque main et de tirer au hasard sur les passants, qui définit l'acte surréaliste, selon l'un des premiers textes de Breton) $)^{45}$.

Sartre sentait parfaitement qu'il fallait dépasser la révolte surréaliste, et souhaitait l'émergence d'une littérature qui fût à la fois négation et construction :

La littérature concrète sera synthèse de la Négativité, comme pouvoir d'arrachement au donné, et du Projet, comme esquisse d'un ordre futur ${ }^{46}$.

Mais dans les années 1950, il remit le «Projet » entre les mains du PC, seul médiateur autorisé du changement révolutionnaire, pariant sur le fait que le parti sorte un jour du moment totalitaire dans lequel il se trouvait, car «la négativité du parti communiste [contrairement à la négativité surréaliste] est provisoire, c'est un moment historique nécessaire dans sa grande entreprise de réorganisation sociale $»^{47}$. La profession de foi allait ainsi justifier plusieurs années de compagnonnage avec le communisme, un lien qui ne commença à se détendre qu'à partir de la répression soviétique en Hongrie en 1956. Mais, en dépit de tout, la foi révolutionnaire demeurait intacte.

$\mathrm{Au}$ terme de sa critique de l'engagement sartrien, Rougemont concluait son essai en définissant trois figures-types d'écrivains - le ludion, le contestataire et le prophète correspondant à trois modes de relations avec leur temps. Si le ludion, «immergé dans l'époque", était capable d'en reconstituer l'atmosphère particulière, il restait néanmoins "passif», il ne cherchait pas à peser sur l'événement, ne faisant qu'enregistrer son écho. Le contestataire, comme son nom l'indique, « réagit contre l'époque ", mais son refus, pour "dévastant " et «impitoyable » qu'il soit, s'avérait rapidement inefficace faute d'un projet alternatif à promouvoir. Face à ces deux figures se dressait le prophète, c'est-à-dire celui qui « sent l'époque (bien mieux que le ludion) dans la mesure où il la refuse (bien plus radicalement que le contestataire), mais s'il la juge et la refuse, c'est au nom d'une vision meilleure qu'il annonce, illustre, anticipe... $»^{48}$. Rougemont marquait nettement sa préférence pour cette troisième catégorie, incarnée par des auteurs aussi divers que Thomas More, Hölderlin, Proudhon, Orwell ou Teilhard de Chardin. Avant eux domine la référence aux grands prophètes de la tradition biblique, tels Esaïe ou Jérémie ${ }^{49}$.

\section{Le soutien aux écrivains persécutés}

"S'engager, ce n'est pas prêter son nom ou son autorité », affirmait Rougemont. "Ce n'est pas signer à gauche plutôt qu'à droite, ni même écrire des manifestes en faveur des victimes d'un régime et au nom d'un régime opposé qui ferait pire s'il le pouvait " ${ }^{50}$. En dépit de cette conception à la fois exigeante et circonscrite de l'engagement, ramenant constamment au cœur du métier de l'écrivain, à sa pensée et à son style, force est de constater que Rougemont n'a pas manqué de "prêter son nom ou son autorité » et d'apporter son soutien aux victimes des régimes autoritaires et répressifs 
par des pétitions, des télégrammes, des communiqués de presse, des manifestes de toutes sortes. Ce fut le cas au Congrès pour la liberté de la culture, ce fut encore le cas au PEN qui voyait d'ailleurs dans les campagnes qu'il menait en faveur des écrivains persécutés l'une de ses raisons d'être. Si de telles actions ne prouvaient rien quant à la qualité d'engagement de celles et ceux qui s'y prêtaient, elles témoignaient au moins d'une solidarité avec des écrivains qui eux, étaient réellement engagés, à leurs risques et périls. Comme le rappelait Rougemont dans son essai de 1973, les intellectuels dissidents qui contestaient le régime soviétique méritaient pleinement ce qualificatif :

$\mathrm{Nul}$ art véritable n'a jamais pu se nourrir de mensonge. Nulle tyrannie n'a jamais inspiré une seule grande œuvre à sa louange, et «l'inconditionnel » n'admet que l'uniforme, l'alignement mécanique dans l'informe. D'où l'accusation de " formalisme » que le pouvoir totalitaire a coutume de porter contre tout écrivain qui s'obstine à rester responsable de la vérité de son art. Mais chacun peut le voir aujourd'hui : ce ne sont pas les communistes bon teint de l'Union des écrivains soviétiques qui sont «engagés " par leur œuvre, mais Soljenitsyne qu'ils excluent, Siniavsky et Daniel qu'ils laissent emprisonner sans protester ${ }^{51}$.

De ce point de vue, le centre suisse romand n'hésita pas à monter en première ligne lorsqu'il s'agit de contester l'adhésion au PEN des écrivains soviétiques. La question, qui resurgit périodiquement, s'est posée à nouveau de façon concrète à partir de 1973 quand se déroula, à Helsinki, puis à Genève, la Conférence sur la sécurité et la coopération en Europe, laquelle marquait sur le plan diplomatique une phase de détente dans les relations entre les camps occidental et communiste. Dans ce contexte, le 12 décembre 1973, au comité exécutif du PEN à Londres, une proposition fut faite par les délégués de Hongrie, de Bulgarie et de RDA, concernant l'admission de l'Union des écrivains soviétiques. La proposition était assortie d'une demande de révision de la charte du PEN, car, contrairement à la COMES, les statuts du PEN interdisaient que des délégués de syndicats professionnels d'écrivains pussent adhérer en tant que tels à l'association. Heinrich Böll, alors président du PEN International, répondit qu'il appartenait aux écrivains d'adhérer eux-mêmes, et non en tant que membres de l'Union des écrivains soviétiques, et qu'il était hors de question de procéder à une révision de la charte, une position que ne manquèrent pas d'appuyer le centre suisse romand, celui de Hollande et celui de Suède dans une déclaration commune publiée en $1974^{52}$. Pour les membres du centre suisse romand, la proposition des centres hongrois, bulgares et allemands de l'Est n'était qu'une manœuvre des Soviétiques pour provoquer une scission au sein du PEN International ${ }^{53}$. Rougemont lui-même remettait en question le principe d'un dialogue avec les écrivains soviétiques, alors que la détente au niveau diplomatique ne se traduisait nullement, selon lui, par des mesures concrètes en faveur de la liberté d'expression en URSS. C'est ce qui ressort d'une intervention faite probablement à l'Assemblée générale du centre suisse romand en octobre 1973 :

Il est scandaleux et honteux d'engager un dialogue courtois avec des flics, coupables

d'avoir écrasé la liberté d'expression dans leur pays, au moment où l'œuvre et la vie même des plus grands écrivains de ce pays sont menacés par le pouvoir politique. Il $\mathrm{y}$ a là une indécence profonde, que je n'ai pas à qualifier ${ }^{54}$.

Rougemont soulignait régulièrement la contradiction entre la volonté officielle de dialogue des Soviétiques au niveau international et le sort réservé en URSS aux voix dissidentes. En août 1973, il adressait à Nikolaï Podgorny, président du præsidium du Soviet suprême, une lettre au nom du centre suisse romand dans laquelle il demandait la libération de l'écrivain Andreï Amalrik, toujours détenu en prison alors qu'il venait de purger sa peine : 
À nos yeux, comme aux yeux de l'opinion publique mondiale, le nouvel emprisonnement d'Amalrik infligerait un cinglant démenti à la politique de détente et de sécurité que nous approuvons tous, et que vous déclarez défendre. Il n'y aura pas de détente possible entre les peuples, ni de sécurité pour les habitants de notre continent, tant que la libre expression des opinions d'un citoyen sera considérée comme un crime par le gouvernement de l'un des pays membres de la Conférence ${ }^{55}$. Nous vous demandons, en vertu de notre totale solidarité avec tous les esprits libres et pacifiques, au nom de notre sympathie pour le peuple russe, au nom des principes mêmes que vous affirmez officiellement, et en application de vos propres lois, la libération immédiate d'Amalrik ${ }^{56}$.

Ce témoignage est loin d'être isolé et montre que la lutte en faveur des dissidents en URSS apparaît clairement comme une priorité à l'époque, même si le PEN s'est aussi préoccupé d'aider les écrivains persécutés dans des pays non communistes, en Espagne, en Corée du Sud, en Argentine, en Malaisie notamment, comme le montre ci-après la liste des interventions du centre suisse romand entre 1973 et $1977^{57}$.

- 1973 : En juin, intervention pour alerter sur la discrimination que subissait la langue catalane. En août, intervention en faveur du dissident soviétique Andreï Amalrik.

- 1974 : Télégramme à l'ambassade de Corée du Sud à Berne demandant la libération du poète et journaliste Kim Ji-ha, emprisonné sous prétexte de propagande communiste.

- 1974-1976: Intervention coordonnée avec les centres du PEN yougoslave en faveur d'écrivains croates, dont Mihajlo Mihajlov, jugé pour propagande hostile à l'encontre du gouvernement de Tito et condamné à plusieurs années de prison.

- 1976: Nouvelle intervention pour la libération de Kim Ji-ha, puis du dissident soviétique Vladimir Boukovski.

- 1977 : Soutien à Antonio di Benedetto, journaliste argentin arrêté au début de la dictature militaire ; à Said Zahari, journaliste malaisien détenu depuis 1963 dans les prisons de Singapour sans motif d'inculpation (officieusement pour activités communistes); et à plusieurs reprises en faveur d'écrivains emprisonnés en Uruguay, en Algérie et en Allemagne de l'Est.

Au milieu des années 1970, ce type d'activité apparaissait à Rougemont comme la raison d'être essentielle du PEN :

Laissons à la SSE son rôle d'organisation syndicale des écrivains ; laissons au groupe d'olten son rôle de contestation de la SSE; laissons à l'Alliance culturelle romande son rôle d'animation des échanges régionaux : nous faisons tous partie de l'une au moins de ces associations, et si nous sommes en plus du PEN, cela signifie quoi? Non pas que ces associations sont insuffisantes, mais que d'autres problèmes nous requièrent également, au-delà de notre région, en Europe, en Indonésie, au Chili, en URSS, en Iran... Le plus grave est celui de la persécution des écrivains dans le monde entier. (Pas le seul, il existe des problèmes plus spécifiquement littéraires, mais celui-là est le plus impossible à ignorer $)^{58}$.

Outre les manifestations ponctuelles de soutien, quels étaient les moyens dont disposaient Rougemont et le PEN pour venir en aide aux écrivains persécutés ? Plusieurs organismes existaient, dont les objectifs et les champs d'action étaient complémentaires.

Depuis 1960, il existait au sein du PEN, nous l'avons noté en introduction, un comité des écrivains en prison. Lors de la réunion du comité exécutif du PEN International à Berlin en 1972, une commission fut nommée pour en encadrer l'action, ainsi que pour faire la liaison avec le PEN Emergency Fund, créé pour sa part en janvier 1971 dans le but de venir en aide aux écrivains sortis de prison et à leurs familles. Siégeant en Hollande, le 
comité de ce fonds d'urgence était composé notamment de Denis de Rougemont et d'Heinrich Böll, qui lui versa $10 \%$ de son prix Nobel de littérature obtenu en $1972^{59}$. Depuis lors, cette cagnotte vivait essentiellement des contributions des membres du PEN ou de subventions de tiers. En 1976, une intervention de Rougemont permit de maintenir le budget à flot grâce à un versement de la Fondation européenne de la culture, que l'écrivain avait créée dans les années 1950. Selon le rapport de l'année 1976, plus de 50 écrivains et leurs familles bénéficièrent d'aides matérielles ${ }^{60}$. Ces deux organismes n'agissaient pas exactement sur le même plan : la commission travaillait officiellement au niveau diplomatique, tandis que le fonds d'urgence œuvrait davantage dans la clandestinité en apportant un secours matériel à des écrivains qui, étant sortis de prison, n'étaient plus inscrits sur les listes d'Amnesty International ${ }^{61}$, mais qui vivaient toujours dans une grande précarité.

\section{Conclusion} difficile de juger les conséquences de ces démarches :

Cependant, les écrivains hongrois, par exemple, ont témoigné à maintes reprises du résultat bénéfique pour eux-mêmes de protestations semblables ; savoir, sentir que d'autres écrivains s'occupent d'eux a permis à plusieurs de survivre ${ }^{62}$.

Sans doute Rougemont faisait-il ici allusion à la mobilisation, massive, du Congrès pour la liberté de la culture lors de la répression de la révolte hongroise de 1956. De fait, on ne saurait sous-estimer l'importance psychologique, pour les écrivains qui en furent les bénéficiaires, de ces manifestations de solidarité internationale. Si leur sort dépendait avant tout de l'évolution politique des régimes en place, il était capital pour eux de se sentir soutenus à distance par leurs pairs ${ }^{63}$.

En 1977, Rougemont démissionna de ses fonctions de président du centre suisse romand. Il demeura toutefois son président d'honneur et continua à prêter son nom à des initiatives qui s'inscrivaient dans le même esprit que celles du PEN, comme le montre par exemple son adhésion au Comité international pour le soutien de la Charte 77. Face à la «normalisation » de la société tchécoslovaque, la solidarité à l'égard des dissidents comme Jan Patočka ou Vaclav Havel n'était rien moins que naturelle. Certes, nous l'avons noté, l'écrivain engagé, selon Rougemont, devait être idéalement porteur d'un nouvel ordre politique par le contenu comme par la forme de ses écrits. Mais une telle activité ne pouvait se déployer pleinement que dans une société libre. Ceci nous ramène à la figure-type du "contestataire", dans sa plus noble acception, telle que Rougemont l'avait définie dans son essai sur la responsabilité de l'écrivain :

La contestation la plus féconde et la plus efficace en fin de compte est celle qui oppose à la société aliénante, à l'oppression dégradante, à l'injustice et au mensonge organisé, le témoignage de l'amour, de la fraternité et de l'honneur d'être homme : Silone, Koestler, Malraux, Soljenitsyne, dans la descendance du plus grand de tous, Dostoïevski ${ }^{64}$.

S'il avait écrit son essai quelques années plus tard, Rougemont aurait sans doute rajouté à cette liste le nom de Patočka, victime le 13 mars 1977 d'une hémorragie cérébrale à l'issue d'un interrogatoire policier particulièrement éprouvant. Par les risques qu'il encourait, par sa qualité de témoin, par son destin tragique, Patočka incarna lui aussi, à sa manière, un modèle d'engagement. 


\section{BIBLIOGRAPHIE}

BENDA, Julien, La trahison des clercs, Paris, Grasset, 2003 (1927).

BÖNI, Otto, BOULANGER, MOusse, IMER, André, LOETSCHER, Hugo, NIEDERER, Ueli, Écrire pour vivre : histoire de la Société suisse des écrivains, Aarau, Éditions Sauerländer, 1987.

CHENU, Roselyne, En lutte contre les dictatures. Le Congrès pour la liberté de la culture (1950-1978), entretiens avec Nicolas Stenger, préface d'Alfred Grosser, Paris, Éditions du Félin, 2018.

DENIS, Benoît, Littérature et engagement, de Pascal à Sartre, Paris, Éditions du Seuil, 2000.

MUEHLETHALER, Hans, Die Gruppe Olten. Das Erbe einer rebellierenden Schriftstellergeneration, Aarau, Verlag Sauerländer, 1989.

Prost, Antoine, «Changer le siècle », Vingtième Siècle, 60 (décembre 1998), p. 14-26.

RACINE, Nicole, «L'action européenne des PEN Clubs de 1945 aux années soixante », in Andrée Bachoud, Josefina Cuesta, Michel Trebitsch (dir.), Les intellectuels et l'Europe de 1945 à nos jours, Paris, Publications universitaires Denis Diderot, 2000, p. 103-120.

-, «Associations internationales d'écrivains dans la guerre froide : l'exemple du PEN Club français », in Antoine Fleury, Lubor Jílek (dir.), Une Europe malgré tout, 1945-1990. Contacts et réseaux culturels, intellectuels et scientifiques entre Européens dans la guerre froide, Bruxelles, Peter Lang, 2009, p. 139-151.

-, « La COMES (1958-1969) : une association d'écrivains dans la guerre froide », in Jean-François Sirinelli, Georges-Henri Soutou (dir.), Culture et guerre froide, Paris, Presses de l'Université ParisSorbonne, 2008, p. 281-300.

Rougemont, Denis de, «Confession tendancieuse », Les Cahiers du Mois, 21-22, III -IV cahiers (1926), p. 144-148.

—, « Adieu, beau désordre... », Bibliothèque universelle et Revue de Genève, (mars 1926), p. 311-319.

-, Le paysan du Danube, Lausanne, Payot, 1932.

-, « Préface à une littérature », Esprit, 25 (octobre 1934), p. 24-33.

-, Penser avec les mains, Paris, Albin Michel, 1936.

-, « M. Benda nous “ cherche ” mais ne nous trouve pas », Esprit, 58 ( $1^{\text {er }}$ juillet 1937), p. 616-618.

-, « Vers une littérature personnaliste », À Nous la Liberté, 4 (27 mars 1937), p. 10.

—, « Trop d'irresponsables s'engagent! (Responsabilité des intellectuels) », L'Ordre Nouveau, 42 (juin 1938), p. 19-22.

_, Fédéralisme culturel, Neuchâtel, Éditions de la Baconnière, 1965.

-, Responsabilité de l'écrivain dans la société européenne d'aujourd'hui / Die Verantwortung des Schriftstellers in der europäischen Gesellschaft der Gegenwart, Zurich, Pro Helvetia, 1973. Traduction anglaise : "The Social Responsibility of the Writer in Contemporary West European Societies ", The Yearbook of Comparative and General Literature, 22 (1973), p. 58-70

_, L'avenir est notre affaire, Paris, Stock, 1977.

_, Préface à Bertrand de LAUNAY, Le poker nucléaire : comme brebis à l'abattoir, Paris, Syros, 1983, p. 9-15. 
SARTRE, Jean-Paul, « Qu'est-ce que la littérature ? », Situations II, Paris, Gallimard, 1948.

—, « Le fantôme de Staline » [1957], Situations VII, Paris, Gallimard, 1965, p. 144-307.

SCHWAMM, Henri, RESZLER, André (éds), Denis de Rougemont, l'écrivain, l'Européen, Neuchâtel, La Baconnière, 1976.

STENGER Nicolas, Denis de Rougemont. Les intellectuels et l'Europe au XX ${ }^{e}$ siècle, Rennes, Presses universitaires de Rennes, 2015.

\section{NOTES}

1. L'essentiel de la littérature sur ce sujet se résume aux travaux de Nicole Racine. Cf. par exemple: «L'action européenne des PEN Clubs de 1945 aux années soixante » et «Associations internationales d'écrivains dans la guerre froide ».

2. N. Racine, «L'action européenne des PEN Clubs de 1945 aux années soixante », p. 108.

3. D. de Rougemont, « 5 ans de PEN S. R.", manuscrit sans date. Bibliothèque publique et universitaire de Neuchâtel, Fonds Denis de Rougemont (désormais BPUN, Fonds DdR), «PEN-1 », ID3081. L'argument est repris de Denis de Rougemont, Fédéralisme culturel, p. 16 sq.

4. Sur l'engagement de Rougemont dans cette association, voir notre livre Denis de Rougemont, chap. XII, XV et XVIII.

5. Sur l'engagement de Pierre Emmanuel, qui fut par ailleurs le président du PEN International entre 1969 et 1971, voir notre livre d'entretiens (En lutte contre les dictatures) avec Roselyne Chenu, qui fut sa principale collaboratrice au Congrès entre 1965 et 1975.

6. Lettre de Denis de Rougemont à Pierre Emmanuel, le 26 mai 1970 (BPUN, Fonds DdR, « Congrès pour la liberté de la culture », ID3069).

7. On trouve dans les archives un dossier de correspondance sur ce sujet, comprenant les lettres de soutien de Louis Armand, Pierre Emmanuel, Henri Brugmans, Ignazio Silone, C. J. Burckardt, Maurice Zermatten, Jean Starobinski, Eugène Ionesco, Lawrence Durrell, Stephen Spender, Albert Gyergyai, Virgil Candea, Marshall McLuhan et Léopold Sédar Senghor. Le prix Nobel de littérature fut attribué en 1971 à Pablo Neruda (BPUN, Fonds DdR, « PEN », ID3081).

8. Lettre de Suzanne Deriex à Denis de Rougemont, le 30 avril 1970 (BPUN, Fonds DdR, « PEN-3 », ID3081).

9. O. Böni et al., Écrire pour vivre.

10. H. Muehlethaler, Die Gruppe Olten.

11. N. Racine, « La COMES (1958-1969)», p. 281 sq.

12. Ces projets n'aboutirent pas et laissèrent Denis de Rougemont pour le moins sceptique, nous y reviendrons.

13. Procès-verbal de l'Assemblée générale du centre suisse romand du PEN, tenue à l'Institut national genevois, le 24 avril 1971 (BPUN, Fonds DdR, « PEN-2 », ID3081).

14. Procès-verbal de l'Assemblée générale du centre suisse romand du PEN, tenue au Foyer de la Maison Pulliérane à Pully, le 30 septembre 1972 (BPUN, Fonds DdR, « PEN-2 », ID3081).

15. Lettre circulaire adressée aux membres du centre suisse romand du PEN par Denis de Rougemont et Suzanne Deriex, avril 1972 (BPUN, Fonds DdR, « PEN-2 », ID3081).

16. Nouveaux membres du centre suisse romand du PEN, 1971-1974 (BPUN, Fonds DdR, « PEN-2 », ID3081).

17. Lettre de Jacques Mercanton à Suzanne Deriex, le 11 mars 1971 (BPUN, Fonds DdR, « PEN-5 », ID3081).

18. Lettre d'Heinrich Böll à Werner Weber, le 10 juillet 1973 (BPUN, Fonds DdR, «PEN-5 », ID3081). 
19. Il existait des centres PEN à Zurich, à Bâle, ainsi qu'à Winterthour. Pour le centre suisse romand, l'enjeu était surtout financier : joindre ses efforts avec ceux des autres PEN en Suisse devait lui permettre d'obtenir plus facilement des subventions de Pro Helvetia.

20. Voir sur ce point la communication de Daniel Rothenbühler, actuel président du centre suisse-allemand du PEN, lors du 70 e anniversaire du PEN suisse romand, le 14 avril 2019, au Centre Dürrenmatt de Neuchâtel.

21. Procès-verbal de l'Assemblée générale du centre suisse romand du PEN, tenue au château de Coppet, le 13 octobre 1973 (BPUN, Fonds DdR, « PEN-2 », ID3081).

22. Participation de la Suisse romande aux comités exécutifs en congrès du PEN International (BPUN, Fonds DdR, « PEN-2 », ID3081).

23. Activités internationales - Bilan 1973-1974 (PEN Club Suisse romande, notes pour rapport du président) (BPUN, Fonds DdR, « PEN-2 », ID3081).

24. Voir la bibliographie, pour la version anglaise.

25. Pour de plus amples développements, voir notre livre Denis de Rougemont, chap. I. Texte accessible en ligne : <http://www.mythe-imaginaire-societe.fr/?p=4564>.

26. J. Benda, La trahison des clercs, p. 132.

27. On trouve les termes de ce débat in D. de Rougemont, «M. Benda nous " cherche" mais ne nous trouve pas». Benda répondit à Rougemont dans une lettre du 12 novembre 1937 (BPUN, Fonds DdR, «Correspondance générale », ID1472; publiée in Denis de Rougemont, l'écrivain, l'Européen, p. 303 sq.).

28. D. de Rougemont, «Confession tendancieuse », p. 144.

29. Id., Le paysan du Danube, p.142.

30. Id., « Vers une littérature personnaliste », p. 10.

31. Id., Penser avec les mains, p. 230-231. Cité dans Denis de Rougemont, Responsabilité de l'écrivain, p. 15 sq.

32. Id., " Adieu, beau désordre... », p. 316.

33. D. de Rougemont, « Trop d'irresponsables s'engagent! », p. 21. Cité avec quelques variantes in Responsabilité de l'écrivain, p. 14 sq.

34. Ibid., p. 4.

35. Ibid. Rougemont entend par là " l'aménagement et l'arbitrage des rapports humains dans la Cité (la polis grecque donne politique, la civitas romaine, civisme) ».

36. Ibid., p. 5.

37. Ibid., p. 6-8.

38. B. Denis, Littérature et engagement, de Pascal à Sartre, p. 61.

39. D. de Rougemont, Responsabilité de l'écrivain, p. 18.

40. J.-P. Sartre, « Qu'est-ce que la littérature ?», p. 277.

41. D. de Rougemont, « Préface à une littérature », p. 28 et 30.

42. A. Prost, « Changer le siècle », p. 22.

43. D. de Rougemont, Responsabilité de l'écrivain, p. 2.

44. J.-P. Sartre, « Le fantôme de Staline », p. 146.

45. D. de Rougemont, Responsabilité de l'écrivain, p. 10.

46. J.-P. Sartre, «Qu'est-ce que la littérature?», p. 107 sq.

47. J.-P. Sartre, «Qu'est-ce que la littérature ?», p. 196 et 225.

48. D. de Rougemont, Responsabilité de l'écrivain, p. 12-14.

49. Voir par exemple Denis de Rougemont, L'avenir est notre affaire, p. 370, et la préface à Bertrand de Launay, Le poker nucléaire, p. 15.

50. D. de Rougemont, Responsabilité de l'écrivain, p. 15.

51. Ibid., p. 17.

52. Déclaration conjointe des centres de Hollande, de Suisse romande et de Suède au comité exécutif du PEN International, 1974 (BPUN, Fonds DdR, « PEN-2 », ID3081). 
53. Lettre de Suzanne Deriex à Denis de Rougemont, le 12 février 1974 (BPUN, Fonds DdR, «PEN-3 », ID3081).

54. Denis de Rougemont, Notes manuscrites sans date (BPUN, Fonds DdR, « PEN-1 », ID3081).

55. Il s'agit de la Conférence sur la sécurité et la coopération en Europe.

56. Lettre adressée le 30 août 1973 par M. Denis de Rougemont à M. Nikolaï Podgorny, président du præsidium du Soviet suprême (BPUN, Fonds DdR, « PEN-2 », ID3081).

57. Les archives consultées dans le Fonds Denis de Rougemont de la BPUN nous informent essentiellement pour les années 1973-1977 (BPUN, Fonds DdR, « PEN-2 » et « PEN-3 »).

58. Denis de Rougemont, « 5 ans de PEN S. R. », manuscrit sans date (BPUN, Fonds DdR, « PEN-1 », ID3081).

59. Lettre de Suzanne Deriex à Denis de Rougemont, le 26 novembre 1972 (BPUN, Fonds DdR, « PEN-3 », ID3081).

60. Foundation PEN Emergency Fund. Extrait du rapport d'activité 1976 présenté au comité exécutif de Hambourg en mai 1977 (BPUN, Fonds DdR, « PEN-2 », ID3081).

61. Notons que depuis le 9 mai 1972, Rougemont faisait également partie du comité de patronage de la section suisse d'Amnesty, aux côtés d'Alfred Andersch, Alexandre Berenstein, Rolf R. Bigler, Walther Bringolf, Friedrich Dürrenmatt, Jean Graven, Paul Guggenheim, Heini Hediger, JeanFlavien Lalive, Kurt Marti, Willy Spühler, Hans O. Staub, Michel Virally, Friedrich Traugott Wahlen, Eduard Zellweger. Les archives conservées à la BPUN font essentiellement mention de campagnes concernant des cas d'emprisonnement en URSS et dans les démocraties populaires. Le 15 mai 1973, Rougemont accorde par ailleurs son patronage à une conférence d'information sur la torture donnée à l'initiative du groupe lausannois d'Amnesty. Peu disponible pour assister aux réunions de la section, il est sollicité le 30 avril 1973 par Fritz Roth, représentant le groupe de Berne d'Amnesty, pour rédiger un article concernant l'abolition de la torture mais décline l'invitation en raison d'une surcharge de travail. Dans les années 1970, des groupes thématiques apparaissent. Rougemont lance un appel en novembre 1978 à la création d'un groupe d'artistes romands désirant se joindre aux actions de solidarités d'Amnesty International, avec Jean-Marie Auberson, Michel Bühler, Étienne Delessert et Jean-Luc Bideau (BPUN, Fonds DdR, «Amnesty International », ID3064).

62. Procès-verbal de l'Assemblée générale du centre suisse romand du PEN, tenue au Château de Coppet, le 13 octobre 1973 (BPUN, Fonds DdR, « PEN-2 », ID3081).

63. Ce point de vue nous a été confirmé par Roselyne Chenu, qui a beaucoup fait au sein de la Fondation pour une entraide intellectuelle européenne créée par le Congrès pour la liberté de la culture, afin d'aider les intellectuels d'Europe de l'Est, de Grèce, d'Espagne et du Portugal : R. Chenu, En lutte contre les dictatures.

64. D. de Rougemont, Responsabilité de l'écrivain, p. 13 sq.

\section{RÉSUMÉS}

À partir d'archives inédites déposées dans le Fonds Denis de Rougemont de la Bibliothèque publique et universitaire de Neuchâtel, nous examinons ici la collaboration entre Denis de Rougemont et le PEN Club, dont l'écrivain prit la présidence du centre romand au début des années 1970. L'analyse permet d'éclairer certains enjeux et problèmes qui se posèrent à cette association, ainsi que son activité de soutien aux écrivains persécutés et emprisonnés qui figure 
parmi ses principales raisons d'être. Plus généralement, il s'agit de s'interroger sur la conception de la responsabilité de l'écrivain promue par Denis de Rougemont, conception développée dès les années trente et réactualisée dans le cadre de son engagement au sein de cette association.

\section{AUTEUR}

NICOLAS STENGER

Université de Genève 